\title{
Does second-hand smoke affect semen quality?
}

\author{
Sebastiano La Maestra $\cdot$ Silvio De Flora $\cdot$ \\ Rosanna T. Micale
}

Received: 3 April 2014 / Accepted: 8 April 2014 / Published online: 18 May 2014

(C) Springer-Verlag Berlin Heidelberg 2014

Environmental cigarette smoke (ECS), also known as second-hand smoke, is an air-diluted mixture of the mainstream cigarette smoke (MCS) exhaled from active smokers and sidestream cigarette smoke (SCS), which may be subjected to aging processes in indoor environments. MCS is the smoke that is drawn and inhaled by active smokers, whereas SCS is the smoke released from the burning cone and through the cigarette paper, mostly between puffs (IARC 2004). Exposure to ECS results in involuntary (or passive) smoking.

It is well documented that active smoking in humans causes a variety of alterations in sperm DNA, such as oxidative DNA damage, DNA adducts, DNA strand breaks, DNA fragmentation, chromosomal aberrations and heritable mutations (Marchetti et al. 2011; Ji et al. 2013) as well as epigenetic alterations, such as DNA methylation (Xu et al. 2013) and dysregulation of microRNA expression (Marczylo et al. 2012). In addition, the adverse effects of active smoking on sperm quality, fertilization ability, reproduction and early development are well established (Practice Committee of the American Society for Reproductive Medicine 2012).

Less information is available about possible effects of involuntary exposures to ECS on sperm genomic alterations, semen quality and fertility. Epidemiological studies on this subject are hampered by the difficulty to assess the extent of exposure to smoke components and biased by the occurrence of several confounding factors.

Using rodent models, we showed that high-dose ECS, composed of $89 \% \mathrm{SCS}$ and $11 \% \mathrm{MCS}$, is able to produce

S. La Maestra $\cdot$ S. De Flora $(\bowtie) \cdot R$. T. Micale

Department of Health Sciences, University of Genoa,

Via A. Pastore 1, 16132 Genoa, Italy

e-mail: sdf@unige.it oxidative stress and DNA damage in sperm cells. In fact, exposure of adult rats to ECS for 4 weeks caused a significant increase in bulky DNA adduct levels in testis (Izzotti et al. 1999). More recently, we showed that exposure of mice to high doses of ECS, starting at birth and continuing until achievement of sexual maturity, causes a variety of interconnected alterations in male gonads, including loss of weight and histopathological alterations of testis and morphological abnormalities of spermatozoa. In addition, oxidative stress was documented by an increase in both reactive oxygen species (ROS) and lipid peroxidation products in sperm cells. Such increase was accompanied by the induction of DNA strand breaks (S. La Maestra, S. De Flora and R.T. Micale, manuscript in preparation). The most important ECS-related abnormality of epididymal spermatozoa was the absence of tail, which is regarded as a sign of sperm fragility and is associated with fertilization failure (Marmor and Grob-Menendez 1991). It should be cautioned that the doses of ECS to which the rodents were exposed in the above studies accounted for a total suspended particulate (TSP) of almost $100 \mathrm{mg} / \mathrm{m}^{3}$ air, which is particularly high and is not comparable to realistic human exposures. In fact, smokers can generate a cloud of smoke accounting for a TSP of 2-4 mg/m $\mathrm{m}^{3}$ air (Hung et al. 2009), and TSP levels have been reported to range from 0.02 to $2 \mathrm{mg} / \mathrm{m}^{3}$ air in indoor environments where people smoke (IARC 2004).

Other experimental studies evaluated sperm DNA quality and reproductive end points after exposing male mice either to MCS or SCS. The results showed that MCS and SCS have differential effects on the genetic integrity and function of sperm and suggested that male exposure to secondhand smoke may affect sperm motility and fertilization rates (Polyzos et al. 2009). Later on, it was found that exposure of mice to SCS did not induce genetic damage in somatic cells but caused tandem repeat (Ms6-hm) mutations in 
sperm cells under conditions producing plasma cotinine levels similar to those observed in passive smokers (Marchetti et al. 2011). Another study used four fertile adult rhesus macaques (Macaca mulatta), which were exposed to ECS for 6 months at an average TSP of $3.7 \mathrm{mg} / \mathrm{m}^{3}$. Under these conditions, ECS did not affect the semen quality and sperm function but it caused metabolic changes detected by NMR in these non-human primates (Hung et al. 2009).

From a mechanistic point of view, smoke metabolites have been shown to cross the blood-seminiferous tubule barrier and to exert a detrimental effect on Sertoli cell secretory function (Kapawa et al. 2004). Although generation of ROS in germ cells is a physiological mechanism required for various functions of spermatozoa, excessive levels are able to attack bases in nucleic acids, amino acid side chains in proteins and double bonds in unsaturated fatty acids (Lü et al. 2010). Oxidative mechanisms are of particular relevance, because the sperm plasma membrane contains high concentrations of polyunsaturated fatty acids, which are highly susceptible to oxidative damage leading to a loss of integrity in the sperm membrane (Aitken et al. 1989). Furthermore, the sperm nucleus lacks protection against oxidative stress and is vulnerable to oxidationmediated DNA damage (Simon and Carrell 2013).

Thus, although the power of epidemiological studies is not sufficient to detect specific alterations, the outcome of experimental studies as well as mechanistic considerations suggest that paternal exposure to second-hand smoke is potentially able to affect the quality of semen. This conclusion adds a further reason of public health concern related to the involuntary exposure to this complex mixture.

\section{References}

Aitken RJ, Clarkson JS, Fishel S (1989) Generation of reactive oxygen species, lipid peroxidation, and human sperm function. Biol Reprod 41:183-197
Hung PH, Froenicke L, Lin CY, Lyons LA, Miller MG, Pinkerton KE, VandeVoort CA (2009) Effects of environmental tobacco smoke in vivo on rhesus monkey semen quality, sperm function, and sperm metabolism. Reprod Toxicol 27:140-148

International Agency for Research on Cancer (2004) IARC monographs on the evaluation of carcinogenic risks to humans. Tobacco smoke and involuntary smoking, Vol. 83. IARC, Lyon

Izzotti A, Bagnasco M, D'Agostini F, Cartiglia C, Lubet RA, Kelloff GJ, De Flora S (1999) Formation and persistence of nucleotide alterations in rats exposed whole-body to environmental cigarette smoke. Carcinogenesis 20:1499-1505

Ji G, Yan L, Wu S, Liu J, Wang L, Zhang S, Shi L, Gu A (2013) Bulky DNA adducts in human sperm associated with semen parameters and sperm DNA fragmentation in infertile men: a cross-sectional study. Environ Health 30:82

Kapawa A, Giannakis D, Tsoukanelis K, Kanakas N, Baltogiannis D, Agapitos E, Loutradis D, Miyagawa I, Sofikitis N (2004) Effects of paternal cigarette smoking on testicular function, sperm fertilizing capacity, embryonic development, and blastocyst capacity for implantation in rats. Andrologia 36:57-68

Lü JM, Lin PH, Yao Q, Chen C (2010) Chemical and molecular mechanisms of antioxidants: experimental approaches and model systems. J Cell Mol Med 14:840-860

Marchetti F, Rowan-Carroll A, Williams A, Polyzos A, Berndt-Weis ML, Yauk CL (2011) Sidestream tobacco smoke is a male germ cell mutagen. Proc Natl Acad Sci U S A 108:12811-12814

Marczylo EL, Amoako AA, Konje JC, Gant TW, Marczylo TH (2012) Smoking induces differential miRNA expression in human spermatozoa: a potential transgenerational epigenetic concern? Epigenetics 7:432-439

Marmor D, Grob-Menendez F (1991) Male infertility due to asthenozoospermia and flagellar anomaly: detection in routine semen analysis. Int J Androl 14:108-116

Polyzos A, Schmid TE, Piña-Guzmán B, Quintanilla-Vega B, Marchetti F (2009) Differential sensitivity of male germ cells to mainstream and sidestream tobacco smoke in the mouse. Toxicol Appl Pharmacol 237:298-305

Practice Committee of the American Society for Reproductive Medicine (2012) Smoking and infertility: a committee opinion. Fertil Steril 98:1400-1406

Simon L, Carrell DT (2013) Sperm DNA damage measured by comet assay. Methods Mol Biol 927:137-146

Xu W, Fang P, Zhu Z, Dai J, Nie D, Chen Z, Qin Q, Wang L, Wang Z, Qiao Z (2013) Cigarette smoking exposure alters pebp1 DNA methylation and protein profile involved in MAPK signaling pathway in mice testis. Biol Reprod 89:1-11 